\title{
关于 Korn 不等式在端点处的一些研究
}

\author{
蒋仁进*，杨晓榕
}

天津大学应用数学中心, 天津 300072

E-mail: rejiang@tju.edu.cn, xryang1007@163.com

收稿日期: 2019-09-25；接受日期：2019-12-12；网络出版日期: 2021-08-09; * 通信作者

国家自然科学基金 (批准号: 11922114, 11671039, 11771043) 资助项目

摘要 设 $\Omega$ 是 $\mathbb{R}^{n}(n \geqslant 2)$ 中的一个有界区域. Korn 不等式是指向量场 $\mathbf{u} \in W^{1, p}\left(\Omega, \mathbb{R}^{n}(1<p<\infty)\right.$ 的梯度矩阵范数会被线性应变张量的范数控制, 然而在端点 $p=1$ 和 $p=\infty$ 处的情形未知. 借助奇 异积分理论, 本文首次给出了在上述端点处关于零边值 Sobolev 向量的 Korn 不等式.

关键词 Korn 不等式 奇异积分 Hardy 空间 BMO 空间

MSC (2020) 主题分类 $35 \mathrm{~A} 23$

\section{1 引言}

设 $\Omega$ 是 $\mathbb{R}^{n}(n \geqslant 2)$ 中的一个有界区域. 令 $1 \leqslant p \leqslant \infty$, 对于任意的向量场 $\mathbf{u}:=\left(u_{1}, u_{2}, \ldots, u_{n}\right) \in$ $W^{1, p}\left(\Omega, \mathbb{R}^{n}\right)$, 记 $D \mathbf{u}$ 和 $\varepsilon(\mathbf{u})$ 分别为 $\mathbf{u}$ 的梯度矩阵和 $D \mathbf{u}$ 的对称部分, 即

$$
D \mathbf{u}:=\left(\frac{\partial u_{i}}{\partial x_{j}}\right)_{1 \leqslant i, j \leqslant n}, \quad \varepsilon(\mathbf{u}):=\left(\varepsilon_{i j}(\mathbf{u})\right)_{1 \leqslant i, j \leqslant n}:=\left(\frac{1}{2}\left(\frac{\partial u_{i}}{\partial x_{j}}+\frac{\partial u_{j}}{\partial x_{i}}\right)\right)_{1 \leqslant i, j \leqslant n} .
$$

Korn 不等式是指如果向量场 $\mathbf{u}$ 满足第一种条件

$$
\mathbf{u}(x)=0, \quad \forall x \in \partial \Omega
$$

或者第二种条件

$$
\int_{\Omega}\left(\frac{\partial u_{i}}{\partial x_{j}}-\frac{\partial u_{j}}{\partial x_{i}}\right) d x=0, \quad 1 \leqslant i, j \leqslant n
$$

则对 $1<p<\infty$, 存在正常数 $C$, 使得

$$
\|D \mathbf{u}\|_{L^{p}(\Omega)} \leqslant C\|\varepsilon(\mathbf{u})\|_{L^{p}(\Omega)} .
$$


注意到为了避免 Korn 不等式 (1.3) 中右端为 0 、而左端不为 0 的情形, 条件 (1.1) 或 (1.2) 是不可或 缺的, 参见文献 $[1,2]$. 该不等式是研究线性弹力方程和流体力学的基本工具, 在偏微分方程等领域中 有许多重要应用, 参见文献 [3-7].

在条件 (1.1) 下, Friedrichs 和 Horgan 分别在文献 [8] 和文献 [9] 中证明了 (1.3) 在任意区域上 都成立. 同时, Kikuchi 和 Oden 在文献 [10] 中说明了常数 $C \leqslant \sqrt{2}$ (最佳常数目前仍是未知的). 在条件 (1.2) 下, Korn 不等式在什么区域上成立引起了广泛关注, 研究取得了显著的进展, 例如: Friedrichs 在文献 [8] 中证明了对 $p=2$, Korn 不等式 (1.3) 在边界只有有限数量的边或角的区域上 成立; Kondratiev 等在文献 [11] 中证明了对 $p=2$, Korn 不等式 (1.3) 在星形域上成立; Ting 在文献 [12] 中证明了对任意 $1 \leqslant p \leqslant \infty$, Korn 不等式 (1.3) 在有界 Lipschitz 区域上成立; 最近, Acosta 等 在 John 区域上建立了 Korn 不等式, Jiang 等进一步证明了在一定条件下 Korn 不等式成立当且仅 当区域是 John 区域, 参见文献 $[3,13]$. 近年来, 关于 Korn 不等式在一些不规则区域上的研究, 例如 Hölder 区域, $s$-John 区域 $(s>1)$, 见文献 [14-16].

注意到以上所有对 Korn 不等式的研究都限制在 $\mathbf{u} \in W^{1, p}\left(\Omega, \mathbb{R}^{n}\right)(1<p<\infty)$ 这个函数空间. 而当 $p=1$ 时, Korn 不等式即使是在一个方体上也不成立, 见 Conti 等在文献 [17] 中给出的反例; 当 $p=\infty$ 时, 通过向量 $\left(y \log \left(x^{2}+y^{2}\right) \phi(x, y),-x \log \left(x^{2}+y^{2}\right) \phi(x, y)\right)$, 其中 $\phi$ 是 $\mathbb{R}^{n}$ 上的光滑函数, 满足 $\operatorname{supp} \phi \subset B(0,2)$, 并且当 $x^{2}+y^{2} \leqslant 1$ 时 $\phi(x, y)=1$, 可以看出 Korn 不等式 (1.3) 在 $p=\infty$ 时 不成立. 自然地, 我们提出如下问题:

向量函数 $\mathbf{u}$ 属于哪一类函数空间使得 Korn 不等式在端点 $p=1$ 或 $p=\infty$ 处成立?

众所周知, 由 Fefferman-Stein 所引入的 Hardy 空间 $H^{1}\left(\mathbb{R}^{n}\right)$ 和 John-Nirenberg 所引入的有界 平均振动空间 $\mathrm{BMO}\left(\mathbb{R}^{n}\right)$ 分别是 Lebesgue 空间 $L^{1}\left(\mathbb{R}^{n}\right)$ 和 $L^{\infty}\left(\mathbb{R}^{n}\right)$ 的一个恰当的替代. 例如, Riesz 变换分别在 $L^{1}\left(\mathbb{R}^{n}\right)$ 和 $L^{\infty}\left(\mathbb{R}^{n}\right)$ 上无界, 但是它在 $H^{1}\left(\mathbb{R}^{n}\right)$ 和 $\mathrm{BMO}\left(\mathbb{R}^{n}\right)$ 上均有界. 受此启发, 在条 件 (1.1) 下, 我们考虑将 Hardy-Sobolev 空间 $H_{z, 0}^{1,1}\left(\Omega, \mathbb{R}^{n}\right.$ ) (见如下定义 2.5) 和 BMO-Sobolev 空间 $\mathrm{BMO}_{z, 0}^{1}\left(\Omega, \mathbb{R}^{n}\right)$ (见如下定义 2.6) 作为研究空间, 借助奇异积分理论, 用区域上的 Hardy 空间 $H_{z}^{1}(\Omega$ ) (见如下定义 2.3) 和有界平均振动空间 $\mathrm{BMO}_{z}(\Omega)$ (见如下定义 2.4) 分别代替 Korn 不等式 (1.3) 中 的 $L^{1}(\Omega)$ 和 $L^{\infty}(\Omega)$, 得到了上面问题的肯定回答. 本文的主要结果可叙述如下:

定理1.1 设 $\Omega$ 是 $\mathbb{R}^{n}$ 中的一个有界区域, 则存在正常数 $C$, 使得对于任意的向量场 $\mathbf{u} \in$ $H_{z, 0}^{1,1}\left(\Omega, \mathbb{R}^{n}\right)$, 有

$$
\|D \mathbf{u}\|_{H_{z}^{1}(\Omega)} \leqslant C\|\varepsilon(\mathbf{u})\|_{H_{z}^{1}(\Omega)} .
$$

定理1.2 设 $\Omega$ 是 $\mathbb{R}^{n}$ 中的一个有界区域, 则存在正常数 $C$, 使得对于任意的向量场 $\mathbf{u} \in$ $\operatorname{BMO}_{z, 0}^{1}\left(\Omega, \mathbb{R}^{n}\right)$, 有

$$
\|D \mathbf{u}\|_{\mathrm{BMO}_{z}(\Omega)} \leqslant C\|\varepsilon(\mathbf{u})\|_{\mathrm{BMO}_{z}(\Omega)} .
$$

事实上, 上述 Korn 不等式将是以下更强结果的推论. 令 $1 \leqslant p \leqslant \infty$, 对于任意的向量场 $\mathbf{u}:=\left(u_{1}, u_{2}, \ldots, u_{n}\right) \in W^{1, p}\left(\Omega, \mathbb{R}^{n}\right)$, 记

$$
S(\mathbf{u}):=\varepsilon(\mathbf{u})-\frac{\operatorname{div}(\mathbf{u})}{n} I_{n \times n} .
$$

称 $S(\mathbf{u})$ 为 $D \mathbf{u}$ 的反保形部分, 参见文献 [18]. 
定理1.3 设 $\Omega$ 是 $\mathbb{R}^{n}$ 中的一个有界区域, 则存在正常数 $C$, 使得对于任意的向量场 $\mathbf{u} \in$ $H_{z, 0}^{1,1}\left(\Omega, \mathbb{R}^{n}\right)$, 有

$$
\|D \mathbf{u}\|_{H_{z}^{1}(\Omega)} \leqslant C\|S(\mathbf{u})\|_{H_{z}^{1}(\Omega)} .
$$

定理1.4 设 $\Omega$ 是 $\mathbb{R}^{n}$ 中的一个有界区域, 则存在正常数 $C$, 使得对于任意的向量场 $\mathbf{u} \in$ $\operatorname{BMO}_{z, 0}^{1}\left(\Omega, \mathbb{R}^{n}\right)$, 有

$$
\|D \mathbf{u}\|_{\mathrm{BMO}_{z}(\Omega)} \leqslant C\|S(\mathbf{u})\|_{B M O_{z}(\Omega)} .
$$

上述不等式 $1<p<\infty$ 的情形可以参考文献 [19-21]. 称定理 1.3 及 1.4 中出现的 Korn 不等式 为共形的 Korn 不等式. 根据文献 [20], 共形的 Korn 不等式在广义相对论中有着重要的应用. 不难 发现, 定理 1.1 及 1.2 的结论可直接由定理 1.3 及 1.4 蕴含, 只需注意到

$$
\|S(\mathbf{u})\|_{H_{z}^{1}(\Omega)} \leqslant C\|\varepsilon(\mathbf{u})\|_{H_{z}^{1}(\Omega)},
$$

以及

$$
\|S(\mathbf{u})\|_{\mathrm{BMO}_{z}(\Omega)} \leqslant C\|\varepsilon(\mathbf{u})\|_{\mathrm{BMO}_{z}(\Omega)} .
$$

本文余下内容的结构如下. 第 2 节回顾了一些文中用到的函数空间的定义和记号. 第 3 节借助 奇异积分理论证明了定理 1.3 和 1.4 .

为行文方便, 对全文做如下约定. 用 $C$ 表示与主要变量无关的正常数, 但它在不同行可表示不 同的值, 而带有下标的正常数则表示固定的常数, 如 $C_{1}$. 记 $\mathcal{S}\left(\mathbb{R}^{n}\right)$ 为 $\mathbb{R}^{n}$ 上的 Schwarz 函数的集合, $\mathcal{D}(\Omega)$ 为在 $\Omega$ 中有紧支集的光滑函数的集合, $\mathcal{D}^{\prime}(\Omega)$ 为 $\mathcal{D}(\Omega)$ 上的分布. 用 $\omega_{n}$ 表示 $\mathbb{R}^{n}$ 中单位球的 表面积.

\section{2 预备知识}

本节首先回顾一些函数空间的定义和表示.

定义2.1 $([22,23])$ 设 $\phi \in \mathcal{D}\left(\mathbb{R}^{n}\right)$ 且满足 $\int_{\mathbb{R}^{n}} \phi(x) d x \neq 0$, 对任意的 $t>0$, 记 $\phi_{t}(\cdot):=t^{-n} \phi(\cdot / t)$. Hardy 空间 $H^{1}\left(\mathbb{R}^{n}\right)$ 定义为

$$
H^{1}\left(\mathbb{R}^{n}\right):=\left\{f \in \mathcal{D}^{\prime}\left(\mathbb{R}^{n}\right): M_{\phi} f \in L^{1}\left(\mathbb{R}^{n}\right)\right\},
$$

其中

$$
M_{\phi} f(x):=\sup _{t>0}\left|\phi_{t} * f(x)\right|:=\sup _{t>0}\left|\int_{\mathbb{R}^{n}} \phi_{t}(x-y) f(y) d y\right|
$$

称为 $f$ 关于 $\phi$ 的径向极大函数. 定义范数

$$
\|f\|_{H^{1}\left(\mathbb{R}^{n}\right)}:=\left\|M_{\phi} f\right\|_{L^{1}\left(\mathbb{R}^{n}\right)} .
$$


定义2.2 ([24,25]) 有界平均振动空间 $\mathrm{BMO}\left(\mathbb{R}^{n}\right)$ 定义为

$$
\operatorname{BMO}\left(\mathbb{R}^{n}\right):=\left\{f \in L_{\mathrm{loc}}^{1}\left(\mathbb{R}^{n}\right):\|f\|_{\mathrm{BMO}\left(\mathbb{R}^{n}\right)}<\infty\right\}
$$

其中

$$
\|f\|_{\mathrm{BMO}\left(\mathbb{R}^{n}\right)}:=\sup _{Q \subset \mathbb{R}^{n}} \frac{1}{|Q|} \int_{Q}\left|f(x)-f_{Q}\right| d x .
$$

这里 $Q$ 为 $\mathbb{R}^{n}$ 中边与坐标轴平行的方体, $|Q|$ 表示 $Q$ 的 Lebesgue 测度, $f_{Q}:=\frac{1}{|Q|} \int_{Q} f(x) d x$ 称为 $f$ 在 $Q$ 上的积分平均.

不难证明, $H^{1}\left(\mathbb{R}^{n}\right) \subset L^{1}\left(\mathbb{R}^{n}\right)$ 且 $L^{\infty}\left(\mathbb{R}^{n}\right) \subset \mathrm{BMO}\left(\mathbb{R}^{n}\right)$, 参见文献 $[24,25]$.

区域 $\Omega$ 上的 Hardy 空间有两种定义方式. 粗略而言, 一种是支集在 $\bar{\Omega}$ 上的 $H^{1}\left(\mathbb{R}^{n}\right)$ 中的元素的 集合, 另一种是把 $H^{1}\left(\mathbb{R}^{n}\right)$ 中的元素限制到区域 $\Omega$ 上. 本文讨论的是第一种类型的区域上的 Hardy 空间, 现回顾其定义.

定义2.3 ([22,23]) 对任意开集 $\Omega \subset \mathbb{R}^{n}$, 区域 $\Omega$ 上的 Hardy 空间 $H_{z}^{1}(\Omega)$ 定义为

$$
H_{z}^{1}(\Omega):=\left\{f \in \mathcal{D}^{\prime}\left(\mathbb{R}^{n}\right): f \in H^{1}\left(\mathbb{R}^{n}\right), \operatorname{supp} f \subset \bar{\Omega}\right\},
$$

定义范数

$$
\|f\|_{H_{z}^{1}(\Omega)}:=\|f\|_{H^{1}\left(\mathbb{R}^{n}\right)} .
$$

需要指出的是, 设 $\mathbf{f}:=\left(f_{1}, f_{2}, \ldots, f_{n}\right)$ 是定义在区域 $\Omega$ 上的 $n$ 维向量, 如果对所有的 $1 \leqslant i \leqslant n$ 都有 $f_{i} \in H_{z}^{1}(\Omega)$, 那么称 $\mathbf{f} \in H_{z}^{1}(\Omega)$ 且

$$
\|\mathbf{f}\|_{H_{z}^{1}(\Omega)}:=\sum_{i=1}^{n}\left\|f_{i}\right\|_{H_{z}^{1}(\Omega)} .
$$

与区域上的 Hardy 空间类似，区域 $\Omega$ 上的 BMO 空间也有两种定义方式. 现回顾其中一种.

定义2.4 ([22]) 对任意开集 $\Omega \subset \mathbb{R}^{n}$, 区域 $\Omega$ 上的有界平均振动空间 $\mathrm{BMO}_{z}(\Omega)$ 定义为

$$
\mathrm{BMO}_{z}(\Omega):=\left\{f \in L_{\mathrm{loc}}^{1}\left(\mathbb{R}^{n}\right): f \in \operatorname{BMO}\left(\mathbb{R}^{n}\right), \operatorname{supp} f \subset \bar{\Omega}\right\},
$$

定义范数

$$
\|f\|_{\mathrm{BMO}_{z}(\Omega)}:=\|f\|_{\mathrm{BMO}\left(\mathbb{R}^{n}\right)}
$$

类似于 Sobolev 空间, Hardy-Sobolev 和 BMO-Sobolev 空间可分别定义如下.

定义2.5 ([23]) 对任意开集 $\Omega \subset \mathbb{R}^{n}$, 区域 $\Omega$ 上的 Hardy-Sobolev 空间 $H_{z, 0}^{1,1}(\Omega)$ 定义为

$$
\mathrm{BMO}_{z, 0}^{1}(\Omega):=\left\{f \in L_{\mathrm{loc}}^{1}\left(\mathbb{R}^{n}\right): \operatorname{supp} f \subset \bar{\Omega}, \nabla f \in H_{z}^{1}(\Omega)\right\}
$$

定义2.6 $([22,23])$ 对任意开集 $\Omega \subset \mathbb{R}^{n}$, 区域 $\Omega$ 上的 BMO-Sobolev 空间 $\mathrm{BMO}_{z, 0}^{1}(\Omega)$ 定义为

$$
\mathrm{BMO}_{z, 0}^{1}(\Omega):=\left\{f \in L_{\text {loc }}^{1}\left(\mathbb{R}^{n}\right): \operatorname{supp} f \subset \bar{\Omega}, \nabla f \in \mathrm{BMO}_{z}(\Omega)\right\} .
$$




\section{3 定理 1.3 和 1.4 的证明}

本节证明定理 1.3 和 1.4. 为此需要如下的概念和引理.

定义3.1 ( $[24,142$ 页 $])$ 设 $K \in L^{1}\left(\mathbb{R}^{n}\right)$, 记 $\hat{K}$ 为其 Fourier 变换, 如果

(i) $|\hat{K}(x)| \leqslant C_{1}, \forall x \in \mathbb{R}^{n}$;

(ii) 对一切 $y \neq 0$, 有

$$
\int_{|x| \geqslant 2|y|}|K(x-y)-K(x)| d x \leqslant C_{1}
$$

则称 $K$ 是一个 Calderón-Zygmund 奇异积分核, 简称 $\mathrm{C}-\mathrm{Z}$ 核, 其中 $C_{1}$ 称为 $K$ 的 C-Z 常数.

引理3.1 ([24]) 对如上定义的 C-Z 核 $K$, 令 $T f:=K * f$, 则以下三个结论等价:

(i) $\|T f\|_{\mathrm{BMO}\left(\mathbb{R}^{n}\right)} \leqslant C\|f\|_{L^{\infty}\left(\mathbb{R}^{n}\right)}$;

(ii) $\|T f\|_{H^{1}\left(\mathbb{R}^{n}\right)} \leqslant C\|f\|_{H^{1}\left(\mathbb{R}^{n}\right)}$;

(iii) $\|T f\|_{\mathrm{BMO}\left(\mathbb{R}^{n}\right)} \leqslant C\|f\|_{\mathrm{BMO}\left(\mathbb{R}^{n}\right)}$.

其中正常数 $C$ 只与 $n$ 和 $C_{1}$ 有关.

引理 3.1 中定义的积分算子 $T$ 称为 Calderón-Zygmund 奇异积分算子, 简称 C-Z 算子.

引理3.2 设 $\phi \in \mathcal{D}\left(\mathbb{R}^{n}\right)$ 是非负递减的径向函数且满足 $\int_{\mathbb{R}^{n}} \phi(x) d x=1$. 对任意的 $f \in$ $W^{1, p}\left(\mathbb{R}^{n}\right)(1 \leqslant p \leqslant \infty)$, 令 $f_{t}(\cdot):=\phi_{t} * f(\cdot)$, 其中, 对任意的 $t>0, \phi_{t}(\cdot):=t^{-n} \phi(\cdot / t)$, 则有如下结论 成立:

(i) 对 $1 \leqslant p<\infty$, 有 $f_{t} \in \mathcal{S}\left(\mathbb{R}^{n}\right)$, 若进一步, $f$ 有紧支集, 则有 $f_{t} \in \mathcal{D}\left(\mathbb{R}^{n}\right)$;

(ii) 对几乎处处的 $x \in \mathbb{R}^{n}$, 有

$$
\lim _{t \rightarrow 0} f_{t}(x)=f(x)
$$

以及

$$
\lim _{t \rightarrow 0} \frac{\partial f_{t}(x)}{\partial x_{j}}=\frac{\partial f(x)}{\partial x_{j}}
$$

(iii) 对 $1 \leqslant p<\infty$, 有

$$
\lim _{t \rightarrow 0}\left\|f_{t}-f\right\|_{L^{p}\left(\mathbb{R}^{n}\right)}=0 ;
$$

(iv) 对 $1 \leqslant p<\infty$ 以及任意的 $1 \leqslant j \leqslant n$, 有

$$
\lim _{t \rightarrow 0}\left\|\frac{\partial f_{t}}{\partial x_{j}}-\frac{\partial f}{\partial x_{j}}\right\|_{L^{p}\left(\mathbb{R}^{n}\right)}=0 .
$$

证明 (i)-(iii) 的证明参见文献 [25]. 现证 (iv). 由 (iii) 可知, 为证 (iv), 只需证对任意的 $1 \leqslant j \leqslant n$, 下述等式成立

$$
\frac{\partial f_{t}(x)}{\partial x_{j}}=\phi_{t} * \frac{\partial f}{\partial x_{j}}(x)
$$

由

$$
f_{t}(x)=\phi_{t} * f(x)=\int_{\mathbb{R}^{n}} \phi_{t}(x-y) f(y) d y,
$$


等式两边同时对 $x$ 的第 $j$ 个变量求偏导, 则

$$
\begin{aligned}
\frac{\partial f_{t}(x)}{\partial x_{j}} & =\frac{\partial}{\partial x_{j}} \int_{\mathbb{R}^{n}} \phi_{t}(x-y) f(y) d y \\
& =\int_{\mathbb{R}^{n}} \frac{\partial}{\partial x_{j}} \phi_{t}(x-y) f(y) d y \\
& =-\int_{\mathbb{R}^{n}} \frac{\partial}{\partial y_{j}} \phi_{t}(x-y) f(y) d y \\
& =\int_{\mathbb{R}^{n}} \phi_{t}(x-y) \frac{\partial}{\partial y_{j}} f(y) d y \\
& =\phi_{t} * \frac{\partial}{\partial x_{j}} f(x) .
\end{aligned}
$$

引理 3.2 证毕.

以下结论为文献 [18, 命题 9 和 10] 的进一步推广, 也是论文结果证明的主要工具. 对于任意的向 量场 $\mathbf{f} \in W_{\mathrm{loc}}^{1,1}\left(\mathbb{R}^{n}, \mathbb{R}^{n}\right)$, 令

$$
S(\mathbf{f}):=\left(S_{i j}(\mathbf{f})\right)_{1 \leqslant i, j \leqslant n}:=\varepsilon(\mathbf{f})-\frac{\operatorname{div}(\mathbf{f})}{n} I_{n \times n} .
$$

值得注意的是, 上述 (3.1) 式中定义的 $S(\mathbf{f})$ 是一个迹零的对称函数矩阵.

命题3.1 对任意向量场 $\mathbf{f} \in W^{1, p}\left(\mathbb{R}^{n}, \mathbb{R}^{n}\right)(1<p<\infty)$, 下述等式

$$
D_{h} \mathbf{f}(x)=C_{n} D_{h} E S(\mathbf{f})(x)
$$

对 a.e. $x \in \mathbb{R}^{n}$ 成立并在 $L^{p}\left(\mathbb{R}^{n}\right)$ 中成立, 其中 $D_{h}$ 表示沿单位向量 $e_{h}$ 方向的弱导数, $C_{n}=\frac{n}{2(n-1) \omega_{n}}$, 且

$$
\begin{aligned}
& D_{h} E S(\mathbf{f})=\left(D_{h}(E S(\mathbf{f}))_{1}, D_{h}(E S(\mathbf{f}))_{2}, \ldots, D_{h}(E S(\mathbf{f}))_{n}\right), \\
& \begin{aligned}
D_{h}(E S(\mathbf{f}))_{k}(x)= & \frac{4 \omega_{n}}{n+2} S_{h k}(\mathbf{f})(x) \\
& \quad+\sum_{i, j=1}^{n} \int_{\mathbb{R}^{n}} D_{h} \gamma_{i j}^{k}(x-y) S_{i j}(\mathbf{f})(y) d y, \quad 1 \leqslant h, k \leqslant n,
\end{aligned} \\
& \gamma_{i j}^{k}(x)=|x|^{-n}\left(\delta_{i k} x_{j}+\delta_{j k} x_{i}-\delta_{i j} x_{k}\right)+|x|^{-n-2}(n-2) x_{i} x_{j} x_{k},
\end{aligned}
$$

$D_{h} \gamma_{i j}^{k}$ 是一个 $\mathrm{C}-\mathrm{Z}$ 核, $\delta_{i j}$ 为 Kronecker delta 函数.

证明 对任意向量场 $\mathbf{f} \in W^{1, \infty}\left(\mathbb{R}^{n}, \mathbb{R}^{n}\right)$, 文献 [18, 命题 9 和 10] 中分别表明

$$
\mathbf{f}=C_{n} E S(\mathbf{f}), \quad E S(\mathbf{f})=\left((E S(\mathbf{f}))_{1},(E S(\mathbf{f}))_{2}, \ldots,(E S(\mathbf{f}))_{n}\right)
$$

在点态意义下成立, 其中

$$
(E S(\mathbf{f}))_{k}(x)=-\sum_{i, j=1}^{n} \int_{\mathbb{R}^{n}} \gamma_{i j}^{k}(x-y) S_{i j}(\mathbf{f})(y) d y, \quad 1 \leqslant k \leqslant n,
$$


并且

$$
D_{h} \mathbf{f}=C_{n} D_{h} E S(\mathbf{f})
$$

在分布意义下成立. 由 Sobolev 函数的定义可知, $C_{n} D_{h} E S(\mathbf{f})$ 即为 $\mathbf{f}$ 沿 $h$ 方向的弱导数, 见文 献 $[26]$.

取光滑函数 $\phi, \operatorname{supp} \phi \subset B(0,1)$, 当 $|x| \leqslant 1 / 2$ 时 $\phi(x)=1$ 且满足 $\int_{\mathbb{R}^{n}} \phi(x) d x=1$. 对任意向量 场 $\mathbf{f} \in W^{1, p}\left(\mathbb{R}^{n}, \mathbb{R}^{n}\right)(1<p<\infty)$, 令 $\mathbf{f}_{t}(\cdot):=\phi_{t} * \mathbf{f}(\cdot)$, 其中, 对任意的 $t>0, \phi_{t}(\cdot):=t^{-n} \phi(\cdot / t)$. 由 引理 3.2(i) 知 $\mathbf{f}_{t} \in \mathcal{S}\left(\mathbb{R}^{n}, \mathbb{R}^{n}\right) \subset W^{1, \infty}\left(\mathbb{R}^{n}, \mathbb{R}^{n}\right)$. 记 $S\left(\mathbf{f}_{t}\right):=\left(S_{i j}\left(\mathbf{f}_{t}\right)\right)_{1 \leqslant i, j \leqslant n}$, 那么对 $\mathbf{f}_{t}$ 有

$$
\mathbf{f}_{t}(x)=C_{n} E S\left(\mathbf{f}_{t}\right)(x),
$$

并且下述等式在分布意义下成立，

$$
D_{h} \mathbf{f}_{\mathbf{t}}=C_{n} D_{h} E S\left(\mathbf{f}_{t}\right)
$$

由

$$
\begin{aligned}
D_{h}\left(E S\left(\mathbf{f}_{t}\right)\right)_{k}(x)= & \frac{4 \omega_{n}}{n+2} S_{h k}\left(\mathbf{f}_{t}\right)(x) \\
& +\sum_{i, j=1}^{n} \int_{\mathbb{R}^{n}} D_{h} \gamma_{i j}^{k}(x-y) S_{i j}\left(\mathbf{f}_{t}\right)(y) d y, \quad 1 \leqslant h, k \leqslant n,
\end{aligned}
$$

以及 $D_{h} \gamma_{i j}^{k}$ 是一个 C-Z 核 (参见文献 $[18$, 命题 10]), 可知

$$
\begin{aligned}
\left\|D_{h}\left(E S\left(\mathbf{f}_{t}\right)\right)_{k}\right\|_{L^{p}\left(\mathbb{R}^{n}\right)} & \leqslant C\left\|S_{h k}\left(\mathbf{f}_{t}\right)\right\|_{L^{p}\left(\mathbb{R}^{n}\right)}+C \sum_{i, j=1}^{n}\left\|S_{i j}\left(\mathbf{f}_{t}\right)\right\|_{L^{p}\left(\mathbb{R}^{n}\right)} \\
& \leqslant C \sum_{i, j=1}^{n}\left\|S_{i j}\left(\mathbf{f}_{t}\right)\right\|_{L^{p}\left(\mathbb{R}^{n}\right)} \\
& \leqslant C \sum_{i, j=1}^{n}\left\|S_{i j}(\mathbf{f})\right\|_{L^{p}\left(\mathbb{R}^{n}\right)} .
\end{aligned}
$$

其中最后一步由引理 3.2 推出.

由上述估计以及 $(3.3)$ 式推知 $\mathbf{f}_{t} \in W^{1, p}\left(\mathbb{R}^{n}, \mathbb{R}^{n}\right)$ 且 $D_{h} \mathbf{f}_{t} \in L^{p}\left(\mathbb{R}^{n}\right)$.

根据奇异积分在 $L^{p}\left(\mathbb{R}^{n}\right)$ 上的有界性, $\mathbf{f}_{t}=\phi_{t} * \mathbf{f}$, 以及引理 3.2 进一步可知, 存在正常数 $C$, 使得

$$
\begin{aligned}
\lim _{t \rightarrow 0} & \left\|D_{h}\left(E S\left(\mathbf{f}_{t}\right)\right)_{k}-D_{h}(E S(\mathbf{f}))_{k}\right\|_{L^{p}\left(\mathbb{R}^{n}\right)} \\
& \leqslant \lim _{t \rightarrow 0}\left\{C\left\|S_{h k}\left(\mathbf{f}_{t}\right)-S_{h k}(\mathbf{f})\right\|_{L^{p}\left(\mathbb{R}^{n}\right)}+C \sum_{i, j=1}^{n}\left\|S_{i j}\left(\mathbf{f}_{t}\right)-S_{i j}(\mathbf{f})\right\|_{L^{p}\left(\mathbb{R}^{n}\right)}\right\} \\
& =0 .
\end{aligned}
$$

由引理 3.2 可知, $D_{h} \mathbf{f}_{\mathbf{t}}$ 几乎处处收玫于 $D_{h} \mathbf{f}$, 并且在 $L^{p}\left(\mathbb{R}^{n}\right)$ 中收玫于 $D_{h} \mathbf{f}$. 进一步, 由此以及 (3.3) 和 (3.4) 式, 推出

$$
D_{h} \mathbf{f}(x)=C_{n} D_{h} E S(\mathbf{f})(x), \quad \text { a.e. } x \in \mathbb{R}^{n}
$$

且在 $L^{p}\left(\mathbb{R}^{n}\right)$ 中成立. 命题 3.1 证毕. 
下面我们给出定理 1.3 的证明.

证明 首先, 对任意向量场 $\mathbf{u} \in H_{z, 0}^{1,1}\left(\Omega, \mathbb{R}^{n}\right)$, 由 $\mathcal{D}(\Omega)$ 在 $H_{z, 0}^{1,1}(\Omega)$ 中稠密 (参见文献 [22]), 可知 存在向量场 $\mathbf{u}_{t} \in \mathcal{D}\left(\Omega, \mathbb{R}^{n}\right)$, 使得

$$
\lim _{t \rightarrow 0}\left\|\mathbf{u}_{t}-\mathbf{u}\right\|_{H_{z, 0}^{1,1}(\Omega)}=0
$$

为应用命题 3.1 , 我们令

$$
\tilde{\mathbf{u}}:= \begin{cases}\mathbf{u}, & x \in \Omega \\ 0, & x \in \Omega^{\complement}\end{cases}
$$

记为 $\tilde{\mathbf{u}}:=\left(\tilde{u}_{1}, \tilde{u}_{2}, \ldots, \tilde{u}_{n}\right)$. 类似地, 令

$$
\tilde{\mathbf{u}}_{t}:= \begin{cases}\mathbf{u}_{t}, & x \in \Omega \\ 0, & x \in \Omega^{\complement}\end{cases}
$$

记为 $\tilde{\mathbf{u}}_{t}:=\left(\tilde{u}_{t}^{1}, \tilde{u}_{t}^{2}, \ldots, \tilde{u}_{t}^{n}\right)$. 由 $D \mathbf{u}$ 的表示以及定义 2.3 和 2.5 , 可知

$$
\lim _{t \rightarrow 0}\left\|D \tilde{\mathbf{u}}_{t}-D \tilde{\mathbf{u}}\right\|_{H^{1}\left(\mathbb{R}^{n}\right)}=0
$$

结合 (3.1) 式, 这进一步暗示了

$$
\lim _{t \rightarrow 0}\left\|S\left(\tilde{\mathbf{u}}_{t}\right)-S(\tilde{\mathbf{u}})\right\|_{H^{1}\left(\mathbb{R}^{n}\right)}=0 .
$$

其次, 记 $S\left(\tilde{\mathbf{u}}_{t}\right):=\left(S_{i j}\left(\tilde{\mathbf{u}}_{t}\right)\right)_{1 \leqslant i, j \leqslant n}$, 应用命题 3.1 可知, 下述等式

$$
D_{h}\left(\tilde{u}_{t}^{k}\right)(x)=\frac{4 \omega_{n} C_{n}}{n+2} S_{h k}\left(\tilde{\mathbf{u}}_{t}\right)(x)+C_{n} \sum_{i, j=1}^{n} \int_{\mathbb{R}^{n}} D_{h} \gamma_{i j}^{k}(x-y) S_{i j}\left(\tilde{\mathbf{u}}_{t}\right)(y) d y, \quad 1 \leqslant h, k \leqslant n
$$

在几乎处处意义下以及 $L^{p}\left(\mathbb{R}^{n}\right)$ 中成立, 其中 $1<p<\infty$, 因为这里 $\tilde{\mathbf{u}}_{t} \in \mathcal{D}\left(\mathbb{R}^{n}, \mathbb{R}^{n}\right) \subset W^{1, p}\left(\mathbb{R}^{n}, \mathbb{R}^{n}\right)$.

因此, 我们有

$$
\begin{aligned}
D_{h}\left(\tilde{u}_{t}^{k}\right)(x)= & C_{n} D_{h}\left(E S\left(\tilde{\mathbf{u}}_{t}\right)\right)_{k}(x) \\
= & \frac{2 n}{(n-1)(n+2)} S_{h k}\left(\tilde{\mathbf{u}}_{t}\right)(x) \\
& +\frac{n}{2(n-1) \omega_{n}} \sum_{i, j=1}^{n} \int_{\mathbb{R}^{n}} D_{h} \gamma_{i j}^{k}(x-y) S_{i j}\left(\tilde{\mathbf{u}}_{t}\right)(y) d y \\
= & C_{2} S_{h k}\left(\tilde{\mathbf{u}}_{t}\right)(x)+C_{3} \sum_{i, j=1}^{n} T^{k} S_{i j}\left(\tilde{\mathbf{u}}_{t}\right)(x),
\end{aligned}
$$

这里

$$
C_{2}=\frac{2 n}{(n-1)(n+2)}, \quad C_{3}=\frac{n}{2(n-1) \omega_{n}},
$$


并且

$$
T^{k} S_{i j}\left(\tilde{\mathbf{u}}_{t}\right)(x):=\int_{\mathbb{R}^{n}} D_{h} \gamma_{i j}^{k}(x-y) S_{i j}\left(\tilde{\mathbf{u}}_{t}\right)(y) d y,
$$

其中 $T^{k}$ 是与 $\tilde{\mathbf{u}}_{t}$ 的第 $k$ 个分量有关的 $\mathrm{C}-\mathrm{Z}$ 算子.

此外, 由 (3.9) 式和引理 3.1(ii) 即得

$$
\left\|T^{k} S_{i j}\left(\tilde{\mathbf{u}}_{t}\right)\right\|_{H^{1}\left(\mathbb{R}^{n}\right)} \leqslant C\left\|S_{i j}\left(\tilde{\mathbf{u}}_{t}\right)\right\|_{H^{1}\left(\mathbb{R}^{n}\right)} .
$$

联立 (3.8) 和 (3.10) 式, 从而知

$$
\begin{aligned}
\left\|D \tilde{\mathbf{u}}_{t}\right\|_{H^{1}\left(\mathbb{R}^{n}\right)} & =\sum_{h, k=1}^{n}\left\|D_{h}\left(\tilde{u}_{t}^{k}\right)\right\|_{H^{1}\left(\mathbb{R}^{n}\right)} \\
& \leqslant \sum_{h, k=1}^{n}\left(C_{2}\left\|S_{h k}\left(\tilde{\mathbf{u}}_{t}\right)\right\|_{H^{1}\left(\mathbb{R}^{n}\right)}+C_{3} \sum_{i, j=1}^{n}\left\|T^{k} S_{i j}\left(\tilde{\mathbf{u}}_{t}\right)\right\|_{H^{1}\left(\mathbb{R}^{n}\right)}\right) \\
& \leqslant \sum_{h, k=1}^{n}\left(C_{2}\left\|S_{h k}\left(\tilde{\mathbf{u}}_{t}\right)\right\|_{H^{1}\left(\mathbb{R}^{n}\right)}+C C_{3} \sum_{i, j=1}^{n}\left\|S_{i j}\left(\tilde{\mathbf{u}}_{t}\right)\right\|_{H^{1}\left(\mathbb{R}^{n}\right)}\right) \\
& \leqslant C\left\|S\left(\tilde{\mathbf{u}}_{t}\right)\right\|_{H^{1}\left(\mathbb{R}^{n}\right)} .
\end{aligned}
$$

由此可得

$$
\begin{aligned}
\|D \tilde{\mathbf{u}}\|_{H^{1}\left(\mathbb{R}^{n}\right)} & \leqslant\left\|D \tilde{\mathbf{u}}-D \tilde{\mathbf{u}}_{t}\right\|_{H^{1}\left(\mathbb{R}^{n}\right)}+\left\|D \tilde{\mathbf{u}}_{t}\right\|_{H^{1}\left(\mathbb{R}^{n}\right)} \\
& \leqslant\left\|D \tilde{\mathbf{u}}-D \tilde{\mathbf{u}}_{t}\right\|_{H^{1}\left(\mathbb{R}^{n}\right)}+C\left\|S\left(\tilde{\mathbf{u}}_{t}\right)\right\|_{H^{1}\left(\mathbb{R}^{n}\right)} \\
& \leqslant\left\|D \tilde{\mathbf{u}}-D \tilde{\mathbf{u}}_{t}\right\|_{H^{1}\left(\mathbb{R}^{n}\right)}+C\left(\left\|S\left(\tilde{\mathbf{u}}_{t}\right)-S(\tilde{\mathbf{u}})\right\|_{H^{1}\left(\mathbb{R}^{n}\right)}+\|S(\tilde{\mathbf{u}})\|_{H^{1}\left(\mathbb{R}^{n}\right)}\right) .
\end{aligned}
$$

最后, 上式两端令 $t \rightarrow 0$, 再结合 (3.6) 和 (3.7) 式推出

$$
\begin{aligned}
\|D \mathbf{u}\|_{H_{z}^{1}(\Omega)} & =\|D \tilde{\mathbf{u}}\|_{H^{1}\left(\mathbb{R}^{n}\right)} \\
& \leqslant C\|S(\tilde{\mathbf{u}})\|_{H^{1}\left(\mathbb{R}^{n}\right)} \\
& =C\|S(\mathbf{u})\|_{H_{z}^{1}(\Omega)} .
\end{aligned}
$$

定理 1.3 得证.

在定理 1.4 的证明之前, 首先回顾 BMO 空间中的一个重要性质, 即著名的 John-Nirenberg 不 等式.

引理3.3 $([24,27]) \quad$ 对每一个 $f \in \operatorname{BMO}\left(\mathbb{R}^{n}\right)$, 有

$$
\sup _{Q \subset \mathbb{R}^{n}} \frac{1}{|Q|} \int_{Q} \mathrm{e}^{\lambda\left|f(x)-f_{Q}\right| /\|f\|_{*}} d x \leqslant C,
$$

其中正常数 $\lambda$ 和 $C$ 只与 $n$ 有关.

值得注意的是, 在 (3.11) 式中, 不妨设 $f_{Q}=0$ 及 $\|f\|_{*}=1$, 从而易知

$$
f \in \operatorname{BMO}\left(\mathbb{R}^{n}\right) \subset L_{\mathrm{loc}}^{p}\left(\mathbb{R}^{n}\right),
$$

其中 $0<p<\infty$.

下面给出定理 1.4 的证明. 
证明 首先, 对任意向量场

$$
\mathbf{u} \in \mathrm{BMO}_{z, 0}^{1}\left(\Omega, \mathbb{R}^{n}\right),
$$

类似于定理 1.3 的讨论, 得到 $\tilde{\mathbf{u}}$ 如 (3.5) 式. 由上述引理以及 $\tilde{\mathbf{u}}$ 有紧支集 $\operatorname{supp} \tilde{\mathbf{u}} \subset \bar{\Omega}$, 易知

$$
\tilde{\mathbf{u}} \in W^{1, p}\left(\mathbb{R}^{n}, \mathbb{R}^{n}\right) \quad(1<p<\infty) .
$$

其次, 记

$$
S(\tilde{\mathbf{u}}):=\left(S_{i j}(\tilde{\mathbf{u}})\right)_{1 \leqslant i, j \leqslant n},
$$

应用命题 3.1 即知, 对 $\tilde{\mathbf{u}}$ 有

$$
\begin{aligned}
D_{h}\left(\tilde{u}_{k}\right)(x)= & \frac{4 \omega_{n} C_{n}}{n+2} S_{h k}(\tilde{\mathbf{u}})(x) \\
& +C_{n} \sum_{i, j=1}^{n} \int_{\mathbb{R}^{n}} D_{h} \gamma_{i j}^{k}(x-y) S_{i j}(\tilde{\mathbf{u}})(y) d y, \quad 1 \leqslant h, k \leqslant n,
\end{aligned}
$$

在几乎处处意义下以及 $L^{p}\left(\mathbb{R}^{n}\right)$ 中成立.

故有

$$
D_{h}\left(\tilde{u}_{k}\right)(x)=C_{2} S_{h k}(\tilde{\mathbf{u}})(x)+C_{3} \sum_{i, j=1}^{n} T^{k} S_{i j}(\tilde{\mathbf{u}})(x),
$$

其中

$$
T^{k} S_{i j}(\tilde{\mathbf{u}})(x)=\int_{\mathbb{R}^{n}} D_{h} \gamma_{i j}^{k}(x-y) S_{i j}(\tilde{\mathbf{u}})(y) d y .
$$

由此及引理 3.1(iii) 即知

$$
\left\|T^{k} S_{i j}(\tilde{\mathbf{u}})\right\|_{\mathrm{BMO}\left(\mathbb{R}^{n}\right)} \leqslant C\left\|S_{i j}(\tilde{\mathbf{u}})\right\|_{\mathrm{BMO}\left(\mathbb{R}^{n}\right)} .
$$

最后, 联立 (3.12) 和 (3.13) 式, 则有

$$
\begin{aligned}
\|D \mathbf{u}\|_{\mathrm{BMO}_{z}(\Omega)} & =\|D \tilde{\mathbf{u}}\|_{\mathrm{BMO}\left(\mathbb{R}^{n}\right)} \\
& =\sum_{h, k=1}^{n}\left\|D_{h}\left(\tilde{u}_{k}\right)\right\|_{\mathrm{BMO}\left(\mathbb{R}^{n}\right)} \\
& \leqslant \sum_{h, k=1}^{n}\left(C_{2}\left\|S_{h k}(\tilde{\mathbf{u}})\right\|_{\mathrm{BMO}\left(\mathbb{R}^{n}\right)}+C_{3} \sum_{i, j=1}^{n}\left\|T^{k} S_{i j}(\tilde{\mathbf{u}})\right\|_{\mathrm{BMO}\left(\mathbb{R}^{n}\right)}\right) \\
& \leqslant \sum_{h, k=1}^{n}\left(C_{2}\left\|S_{h k}(\tilde{\mathbf{u}})\right\|_{\mathrm{BMO}\left(\mathbb{R}^{n}\right)}+C C_{3} \sum_{i, j=1}^{n}\left\|S_{i j}(\tilde{\mathbf{u}})\right\|_{\mathrm{BMO}\left(\mathbb{R}^{n}\right)}\right) \\
& \leqslant C\|S(\tilde{\mathbf{u}})\|_{\mathrm{BMO}_{\left(\mathbb{R}^{n}\right)}} \\
& =C\|S(\mathbf{u})\|_{\mathrm{BMO}_{z}(\Omega) .}
\end{aligned}
$$

定理 1.4 得证. 


\section{参考文献}

1 Korn A. Die Eigenschwingungen eines elastichen Korpers mit ruhender Oberflache. Akad der Wissensch Munich Math-phys Kl Beritche, 1906, 36: 351-401

2 Korn A. Ubereinige Ungleichungen, welche in der Theorie der elastischen und elektrischen Schwingungen eine Rolle spielen. Bulletin Internationale. Cracovie Akademie Umiejet. Classe de sciences mathematiques et naturelles, 1909, $705-724$

3 Acosta G, Durán R G, Muschietti M A. Solutions of the divergence operator on John domains. Adv Math, 2006, 206: $373-401$

4 Brenner S C, Scott L R. The Mathematical Theory of Finite Element Methods. Berlin: Springer-Verlag, 1994

5 Mosolov P P, Mjasnikov V P. A proof of Korn's inequality. Dokl Akad Nauk SSSR, 1971, 201: 36-39

6 Nitsche J A. On Korn's second inequality. RAIRO J Numer Anal, 1981, 15: 237-248

7 Payne L E, Weinberger H F. On Korn's inequality. Arch Rational Mech Anal, 1961, 8: 89-98

8 Friedrichs K O. On the boundary-value problems of the theory of elasticity and Korn's inequality. Ann Math, 1947, 48: $441-471$

9 Horgan C O. Korn's inequalities and their applications in continuum mechanics. SIAM Rev, 1995, 37: 491-511

10 Kikuchi N, Oden J T. Contact Problems in Elasticity: A Study of Variational Inequalities and Finite Element Methods. Studies in Applied Mathematics. Philadelphia: SIAM, 1988

11 Kondratiev V A, OleinikO A. On Korn's inequalities. C R Acad Sci Paris Sér I Math, 1989, 308: 483-487

12 Ting T W. Generalized Korn's inequalities. Tensor, 1972, 25: 295-302

13 Jiang R, Kauranen A. Korn's inequality and John domains. Calc Var Partial Differential Equations, 2017, 56: 109

14 Acosta G, Durán R G, Lombardi A L. Weighted Poincaré and Korn inequalities for Hölder $\alpha$ domains. Math Methods Appl Sci, 2006, 29: 387-400

15 Acosta G, Durán R G, López G F. Korn inequality and divergence operator: Counterexamples and optimality of weighted estimates. Proc Amer Math Soc, 2013, 141: 217-232

16 Jiang R, Kauranen A. Korn inequality on irregular domains. J Math Anal Appl, 2015, 423: 41-59

17 Conti S, Faraco D, Maggi F. A new approach to counterexamples to $L^{1}$ estimates: Korn's inequality, geometric rigidity, and regularity for gradients of separately convex functions. Arch Ration Mech Anal, 2005, 423: 287-300

18 Reimann H M. Ordinary differential equations and quasiconformal mappings. Invent Math, 1976, 33: 247-270

19 Breit D, Cianchi A, Diening L. Trace-free Korn inequalities in Orlicz spaces. SIAM J Math Anal, 2017, 49: 2496-2526

20 Dain S. Generalized Korn's inequality and conformal Killing vectors. Calc Var Partial Differential Equations, 2006, 25: 535-540

21 Reshetnyak Yu G. Estimates for certain differential operators with finite-dimensional kernel. Sibirsk Mat Zh, 1970, 11: $414-428$

22 Auscher P, Russ E, Tchamitchian P. Hardy Sobolev spaces on strongly Lipschitz domains of $\mathbb{R}^{n}$. J Funct Anal, 2005, 218: $54-109$

23 Chen X, Jiang R, Yang D. Hardy and Hardy-Sobolev spaces on strongly Lipschitz domains and some applications. Anal Geom Metr Spaces, 2016, 4: 336-362

24 Fefferman C, Stein E M. $H^{p}$ spaces of several variables. Acta Math, 1972, 129: 137-193

25 Stein E M. Singular Integrals and Differentiability Properties of Functions. Princeton: Princeton University Press, 1970

26 Evans L C, Gariepy R F. Measure Theory and Fine Properties of Functions. Studies in Advanced Mathematics. Boca Raton: CRC Press, 1992

27 John F, Nirenberg L. On functions of bounded mean oscillation. Comm Pure Appl Math, 1961, 14: 415-426 


\section{On Korn's inequality at endpoints}

\section{Renjin Jiang \& Xiaorong Yang}

Abstract Let $\Omega$ be a bounded domain in $\mathbb{R}^{n}(n \geqslant 2)$. The Korn inequality states that the norm of the derivatives of a vector field $\mathbf{u} \in W^{1, p}\left(\Omega, \mathbb{R}^{n}\right)(1<p<\infty)$ is bounded by the norm of the linearized strain tensor, and yet the endpoint cases $p=1$ and $p=\infty$ were left open. In this paper, by using the singular integral theory, we derive the Korn inequality for the Sobolev vector fields with vanishing boundary values at endpoints mentioned above for the first time.

Keywords Korn inequality, singular integral, Hardy space, BMO space

$\operatorname{MSC}(2020) \quad 35 \mathrm{A23}$

doi: 10.1360/SSM-2019-0231 